\title{
Elliptic problem driven by different types of nonlinearities
}

\author{
Debajyoti Choudhuri ${ }^{1}$ (I) and Dušan D. Repovš̌ ${ }^{2 *}$ (D)
}

\author{
"Correspondence: \\ dusan.repovs@guest.arnes.si \\ ${ }^{2}$ Faculty of Education and Faculty of \\ Mathematics and Physics, University \\ of Ljubljana, Ljubljana, 1000, \\ Slovenia \\ Full list of author information is \\ available at the end of the article
}

\begin{abstract}
In this paper we establish the existence and multiplicity of nontrivial solutions to the following problem:

$$
(-\Delta)^{\frac{1}{2}} u+u+\left(\ln |\cdot| *|u|^{2}\right)=f(u)+\mu|u|^{-\gamma-1} u, \quad \text { in } \mathbb{R},
$$

where $\mu>0,(*)$ is the convolution operation between two functions, $0<\gamma<1, f$ is a function with a certain type of growth. We prove the existence of a nontrivial solution at a certain mountain pass level and another ground state solution when the nonlinearity $f$ is of exponential critical growth.
\end{abstract}

MSC: 35R11;35J75; 35J60; 46E35

Keywords: Fractional Laplacian; Ground state solution; Singularity

\section{Introduction}

The main objective of this paper is to establish the existence and multiplicity of nontrivial solutions for the following problem:

$$
(-\Delta)^{\frac{1}{2}} u+u+\left(\ln |\cdot| *|u|^{2}\right)=f(u)+\mu|u|^{-\gamma-1} u \quad \text { in } \mathbb{R}
$$

where $\mu>0,(*)$ is the convolution operation between two functions, $0<\gamma<1$. The fractional Laplacian operator $(-\Delta)^{\frac{1}{2}}$ is defined as

$$
(-\Delta)^{\frac{1}{2}} u(x)=C\left(1, \frac{1}{2}\right) \lim _{\epsilon \rightarrow 0} \int_{\mathbb{R} \backslash(x-\epsilon, x+\epsilon)} \frac{u(x)-u(y)}{|x-y|^{2}} d y \quad \text { for every } x \in \mathbb{R},
$$

where $C\left(1, \frac{1}{2}\right)=\frac{2}{\pi}$ is a normalization constant (cf. Ros, Oton, ANd SERRA [36, Formula A.1]). Here, $f$ is a continuous function with an exponential growth whose primitive is $F(t)=\int_{0}^{t} f(s) d s$. Using subcritical or critical polynomial growth of the function $f$ is quite common in the literature pertaining to problems on elliptic PDEs. However, very few have considered the case when the nonlinear term has an exponential subcritical or a critical growth. To begin with, we first recall that a function $f$ is said to have a subcritical

(c) The Author(s) 2021. This article is licensed under a Creative Commons Attribution 4.0 International License, which permits use, sharing, adaptation, distribution and reproduction in any medium or format, as long as you give appropriate credit to the original author(s) and the source, provide a link to the Creative Commons licence, and indicate if changes were made. The images or other third party material in this article are included in the article's Creative Commons licence, unless indicated otherwise in a credit line to the material. If material is not included in the article's Creative Commons licence and your intended use is not permitted by statutory regulation or exceeds the permitted use, you will need to obtain permission directly from the copyright holder. To view a copy of this licence, visit http://creativecommons.org/licenses/by/4.0/. 
exponential growth at $\infty$ if

$$
\lim _{t \rightarrow \infty} \frac{f(t)}{e^{\beta t^{2}}-1}=0 \quad \text { for every } \beta>0 .
$$

Similarly, $f$ is said to be of critical exponential growth at $\infty$ if there exist $\theta \in(0, \pi]$ and $\beta_{0} \in(0, \theta)$ such that

$$
\lim _{t \rightarrow \infty} \frac{f(t)}{e^{\beta t^{2}}-1}= \begin{cases}0, & \text { for every } \beta>\theta \\ \infty, & \text { for every } \beta<\theta\end{cases}
$$

The following are some hypotheses which are commonly assumed for problems with the Moser-Trudinger inequality (cf. Do Ó ET AL. [19] and FeLMER ET AL. [21]):

$\left(A_{1}\right) f \in C(\mathbb{R}, \mathbb{R}), f(0)=0$, has critical exponential growth and $F(t) \geq 0$ for every $t \in \mathbb{R}$;

(A) $\lim _{|t| \rightarrow 0} \frac{f(t)}{|t|}=0$

$\left(A_{3}\right)$ There exists $L>4$ such that $f(t) t \geq L F(t)>0$ for every $t \in \mathbb{R}$ (this condition is used to verify that a Cerami sequence is bounded in the Sobolev space $H^{\frac{1}{2}}(\mathbb{R})$ );

$\left(A_{4}\right)$ There exist $q>4$ and $C_{q}>\frac{[2(q-2)]^{\frac{q-2}{2}}}{q^{\frac{q}{2}}} \frac{\left(S_{q}\right)^{q}}{r_{0}^{q-2}}$ such that $F(t) \geq C_{q}|t|^{q}$ for every $t \in \mathbb{R}$, where $S_{q}, r_{0}>0$ ( $S_{q}$ will be defined in Lemma 3.12).

We now give a short review of the related results. The problem that inspired us to investigate the current problem is from the paper by BoËr AND Miyagaki [6]. The novelty addressed in this work is due to the presence of a singular term which is difficult to handle since the corresponding energy functional ceases to be $C^{1}$. This poses an extra challenge in applying the mountain pass theorem and other results in variational methods that demand the functional to be $C^{1}$. To add to these difficulties, the logarithmic term poses a great challenge to establishing the existence of a convergent subsequence of a Cerami sequence. The idea of module translations from the paper by Cingolani and WeTH [14] will be used. However, since the norm of the space $X$ (the solution space which will be defined in the next section) is not invariant under translations, a new difficulty arises. The issues pertaining to the exponential term will be explained later.

Problems involving nonlocal operators are important in many fields of science and engineering e.g. optimization, finance, phase transitions, stratified materials crystal dislocations, anomalous materials, semipermeable membranes, flame propagation, water waves, soft thin films, conservation laws, etc. We refer the reader to Caffarelli [8], Di NezzA ET AL. [15], and the references therein. The literature pertaining to problems without the logarithmic Choquard and the singular term is quite vast, and it is impossible to list everything in this paper. A seminal work in the field of singularity driven problems is due to LAZER AND MC KENNA [27]. Thereafter the problems with singularity were studied by many researchers; see Ghanmi and Saoudi [22], Oliva and Petitta [31], Saoudi et AL. [37], and the references therein. Some of the other works that the readers can consult involve the fractional Laplacian operator $(-\Delta)^{s}$ when $2 s<N$ and $s \in(0,1)$ are CHANG AND Wang [10] and Felmer et Al. [21]. We note that Felmer et Al. [21] also studied some properties of the solutions besides regularity. Furthermore, DO Ó ET AL. [18] studied the problem without the singular and the logarithmic term with potentials that vanish at infinity. Some more suggested papers are Autuori And PuCCi [4], CAO [9], Do Ó ET AL. 
[17], Iannizzotto and Squassina [25], Lam and Lu [26], Moser [30], and Pucci et AL. [35].

We now turn our attention to the problems involving Choquard logarithmic term. Some related references are Alves and Figueiredo [3], Cingolani and Jeanjean [13], Cingolani and Weth [14], Du and Weth [20], and Wen et al. [41]. Furthermore, CinGOLANI AND WETH [14] proved the existence of infinitely many distinct solutions and a ground-state solution, with $V: \mathbb{R}^{2} \rightarrow(0, \infty)$ which is continuous and $\mathbb{Z}^{2}$-periodic, $f(u)=b|u|^{p-2} u, b>0$. Since the setting is periodic, the global Palais-Smale condition can fail due to the invariance of the functional under the $\mathbb{Z}^{2}$-translations. To tackle this problem, DU AND WETH [20] proved the existence of a mountain pass solution and a ground state solution for local problem (1.1) but without the singular term in the case when $V(x) \equiv \alpha>0,2<p<4$, and $f(u)=|u|^{p-2} u$. They went on to further prove that if $p \geq 3$, then both the energy levels are the same and provided a characterization for them. Cingolani AND JEANJEAN [13] proved the existence of stationary waves with prescribed norm by considering $\lambda \in \mathbb{R}$. WEN ET AL. [41] considered a nonlinearity with a polynomial growth. Alves AND Figueiredo [3] proved the existence of a ground state solution to problem (1.1) without the singular term but with a nonlinearity of the Moser-Trudinger type. For Choquard problems one can refer to Abdellaoui and Bentifour [1], Biswas and Tiwari [5] Bonheure et al. [7], Goel et al. [23], Guo and Wu [24], Lieb [28], and Panda et AL. [33].

This paper is organized as follows. Section 2 is a quick look at the mathematical background, space description. In Section 3 we describe an application of the fractional Laplacian operator for dimension $N=1$ along with a few auxiliary lemmas. In Section 4 we prove a few auxiliary lemmas and our main result. Finally, we give an Appendix to the proofs of all results which have been used in the proof of the main theorem.

\section{Preliminaries}

This section is devoted to presentation of the most important notations, results, remarks that will be used in our study of problem (1.1) (for the remaining background material, we refer the reader to the comprehensive monograph by PAPAgEORgIOU, RĂDULESCU, AND REPOVŠ [34]), and the statement of our main result.

We begin by defining the Hilbert space

$$
W^{\frac{1}{2}, 2}(\mathbb{R})=H^{\frac{1}{2}}(\mathbb{R})=\left\{u \in L^{2}(\mathbb{R}): \iint_{\mathbb{R} \times \mathbb{R}} \frac{|u(x)-u(y)|^{2}}{|x-y|^{2}} d x d y<\infty\right\},
$$

equipped with the norm

$$
\|u\|^{2}=\|u\|_{2}^{2}+\iint_{\mathbb{R} \times \mathbb{R}} \frac{|u(x)-u(y)|^{2}}{|x-y|^{2}} d x d y=\|u\|_{2}^{2}+[u]_{\frac{1}{2}, 2}^{2} .
$$

We denote the Schwartz class of functions by $\mathcal{S}(\mathbb{R})$. Thus, for any $u \in \mathcal{S}(\mathbb{R})$, the Fourier transform of $(-\Delta)^{\frac{1}{2}} u$ is given by $|\xi| \hat{u}$, where $\hat{u}$ denotes the Fourier transform of $u$. Also, by Proposition 3.6 given in Di Nezza ET AL. [15], we have

$$
\left\|(-\Delta)^{\frac{1}{4}} u\right\|_{2}^{2}=\frac{1}{2 \pi} \iint_{\mathbb{R} \times \mathbb{R}} \frac{(u(x)-u(y))^{2}}{|x-y|^{2}} d x d y \quad \text { for every } u \in H^{\frac{1}{2}}(\mathbb{R}) .
$$

The factor $\frac{1}{2 \pi}$ will be ignored in the paper throughout. 
Next, we define a slightly smaller space that will make the associated energy functional well-defined (cf. STUBвE [40, Lemma 2.1]):

$$
X=\left\{u \in H^{\frac{1}{2}}(\mathbb{R}): \int_{\mathbb{R}} \ln (1+|x|)(u(x))^{2} d x<\infty\right\},
$$

endowed with the norm

$$
\|u\|_{X}^{2}=\|u\|_{2}^{2}+\int_{\mathbb{R}} \ln \left(1+|x|^{2}\right)(u(x))^{2} d x=\|u\|_{2}^{2}+\|u\|_{*}^{2} .
$$

Then $X$ is a Hilbert space as well. We define three auxiliary bilinear forms as follows:

$$
\begin{aligned}
& A(u, v)=\iint_{\mathbb{R} \times \mathbb{R}} \ln (1+|x-y|) u(x) v(y) d x d y, \\
& B(u, v)=\iint_{\mathbb{R} \times \mathbb{R}} \ln \left(1+\frac{1}{|x-y|}\right) u(x) v(y) d x d y, \\
& C(u, v)=A(u, v)-B(u, v)=\iint_{\mathbb{R} \times \mathbb{R}} \ln (|x-y|) u(x) v(y) d x d y .
\end{aligned}
$$

We further define the functionals

$$
U: H^{\frac{1}{2}}(\mathbb{R}) \rightarrow \mathbb{R}, \quad V: L^{4}(\mathbb{R}) \rightarrow \mathbb{R}, \quad W: H^{\frac{1}{2}}(\mathbb{R}) \rightarrow \mathbb{R},
$$

by $U(u)=A\left(u^{2}, u^{2}\right), V(u)=B\left(u^{2}, u^{2}\right), W(u)=C\left(u^{2}, u^{2}\right)$.

Clearly, a combination of the Hardy-Littlewood-Sobolev inequality (HLS) LIEB [29], $0 \leq \ln (1+r) \leq r$, for any $r>0$, leads to the inequality.

$$
|B(u, v)| \leq \iint_{\mathbb{R} \times \mathbb{R}} \frac{1}{|x-y|} u(x) v(y) d x d y \leq C_{0}\|u\|_{2}\|v\|_{2} \quad \text { for every } u, v \in L^{2}(\mathbb{R}),
$$

where $C_{0}$ is an (HLS) constant. Consequently, we also have

$$
|V(u)| \leq C_{0}\|u\|_{4}^{4}, \quad \text { for every } u \in L^{4}(\mathbb{R}) .
$$

A standard property of the logarithmic function is

$$
\ln (1+|x \pm y|) \leq \ln (1+|x|+|y|) \leq \ln (1+|x|)+\ln (1+|y|) \quad \text { for every } x, y \in \mathbb{R} .
$$

Using (2.9) in tandem with the Hölder inequality, we get

$$
\begin{aligned}
A(u v, w z) \leq & \|u\|_{*}\|v\|_{*}\|w\|_{2}\|z\|_{2} \\
& +\|u\|_{2}\|v\|_{2}\|w\|_{*}\|z\|_{*} \quad \text { for every } u, v, w, z \in L^{2}(\mathbb{R}) .
\end{aligned}
$$

The following lemma is the celebrated result of MOSER AND TRUdinger [32]. 
Lemma 2.1 There exists $0<\omega \leq \pi$ such that, for all $\beta \in(0, \omega)$, there exists a constant $C_{\beta}>0$ satisfying

$$
\int_{\mathbb{R}}\left(e^{\beta u^{2}}-1\right) d x \leq C_{\beta}\|u\|_{2}^{2}
$$

for all $u \in H^{\frac{1}{2}}(\mathbb{R})$ with $\left\|(-\Delta)^{\frac{1}{4}} u\right\| \leq 1$.

The associated energy functional

$$
E(u)=\frac{1}{2}\|u\|^{2}+\frac{1}{4} W(u)-\int_{\mathbb{R}} F(u) d x-\frac{\mu}{1-\gamma} \int_{\mathbb{R}}|u|^{1-\gamma} d x
$$

is well-defined due to the lemma above and the space definition. However, the functional is not $C^{1}$, which disallows the use of the basic results of variational analysis. To tackle this, we define the cutoff functional $\tilde{E}$ as follows:

$$
\tilde{E}(u)=\frac{1}{2}\|u\|^{2}+W(u)-\int_{\mathbb{R}} \tilde{G}(u) d x .
$$

Here

$$
\tilde{G}(t)= \begin{cases}\mu|t|^{1-\gamma}+F(t), & \text { if }|t|>\underline{u}_{\mu} \\ \mu \underline{u}_{\mu}^{1-\gamma}+F\left(\underline{u}_{\mu}\right), & \text { if }|t| \leq \underline{u}_{\mu}\end{cases}
$$

where $F(t)=\int_{0}^{t} f(s) d s$ and $\underline{u}_{\mu}$ is a solution to

$$
(-\Delta)^{\frac{1}{2}} u+u+\left(\ln |\cdot| *|u|^{2}\right)=\mu u^{-\gamma}, \quad \text { in } \mathbb{R},
$$

whose existence is guaranteed by Lemma A.2. Moreover, by Lemma A.4 in Appendix, one can establish that, for a range of $\mu$, a solution to (1.1) is such that $u>\underline{u}_{\mu}$ a.e. in $\Omega$.

Furthermore, observe that

$$
\lim _{u \rightarrow \infty} \frac{\tilde{G}(u)}{e^{\beta u^{2}}-1}=0
$$

Also, we have

$$
0<\frac{\tilde{G}(u)}{e^{\beta u^{2}}-1}<\frac{\tilde{G}(u)}{e^{\beta u_{\lambda}^{2}}-1} .
$$

Under the limit $u \rightarrow 0$, we have $\frac{\tilde{G}(u)}{e^{\beta u_{\lambda}^{2}-1}} \rightarrow \frac{\underline{u}_{\lambda}^{1-\gamma}}{e^{\beta u_{\lambda}^{2}-1}}$. For $x \in \Omega$, which satisfy $0<\underline{u}_{\lambda}(x)<M \ll$ 1, we have $\left(\underline{u}_{\lambda}(x)\right)^{2}<\left(\underline{u}_{\lambda}(x)\right)^{1-\gamma}$. Hence $\frac{1}{\beta} \approx \frac{\underline{u}_{\lambda}(x)^{2}}{e^{\beta \underline{u}_{\lambda}(x)^{2}-1}}<\frac{\underline{u}_{\lambda}(x)^{1-\gamma}}{e^{\beta} \underline{\underline{u}}_{\lambda}(x)^{2}-1}$. This implies that for a suitable $C^{\prime}>0$ we have

$$
\tilde{G}(u) \leq C^{\prime}\left(e^{\beta u^{2}}-1\right) \quad \text { a.e. in } \Omega \text {. }
$$


By Lemmas 2.1, 3.1 we can now conclude that

$$
\int_{\mathbb{R}} \tilde{G}(u) d x \leq C^{\prime} \int_{\mathbb{R}}\left(e^{\beta u^{2}}-1\right) d x \leq C_{\beta}\|u\|_{2}^{2} \leq C^{\prime}\|u\|^{2} .
$$

Remark 2.2 Under hypotheses $\left(A_{1}\right)-\left(A_{2}\right)$, there exists, for $q>2, \epsilon>0$, and $\beta>\theta$, a constant $c_{2}>0$ such that

$$
|F(u)| \leq \frac{\epsilon}{2}|u|^{2}+c_{2}|u|^{q}\left(e^{\beta|u|^{2}}-1\right) \quad \text { for every } u \in X
$$

Furthermore, there exists a constant $c_{3}>0$ satisfying the following inequality:

$$
|f(u)| \leq \epsilon|u|+c_{3}|u|^{q-1}\left(e^{\beta|u|^{2}}-1\right) \quad \text { for every } u \in X
$$

An important consequence of (2.15) is the following:

Consider $\rho_{1}, \rho_{2}>1, \rho_{1} \sim 1, \rho_{2}>2$ such that $\frac{1}{\rho_{1}}+\frac{1}{\rho_{2}}=1$. Then it follows that, for any $u \in H^{\frac{1}{2}}(\mathbb{R}), \epsilon>0, \beta>\theta$, we have that

$$
\int_{\mathbb{R}}|F(u)| d x \leq \frac{\epsilon}{2}\|u\|^{2}+c_{2}\|u\|_{\rho_{2} q}^{q}\left(\int_{\mathbb{R}}\left(e^{\rho_{1} \beta u^{2}}-1\right) d x\right)^{\frac{1}{\rho_{1}}}
$$

Remark 2.3 Henceforth,

1. the notation of a subsequence will be the same as its sequence;

2. the notation for cutoff energy functional $\tilde{E}$ will be continued to be denoted by $E$.

We are now in a position to state our main result.

Theorem 2.4 Assume that hypotheses $\left(A_{1}\right)-\left(A_{4}\right)$ are satisfied, and let $q>4$ and $C_{q}>0$ be chosen sufficiently large. Then

(i) problem (1.1) has a solution $u \in X \backslash\{0\}$ such that

$$
\left.E(u)=\inf _{\gamma \in \Gamma \in[0,1]} \max _{t \in(}\{E(\gamma))\right\}=d,
$$

where

$$
\Gamma=\{\gamma \in C([0,1], X): \gamma(0), E(\gamma(1))<0\}
$$

is the class of paths on $X$ joining $\gamma(0)$ and $\gamma(1)$;

(ii) problem (1.1) has a ground state solution $u \in X \backslash\{0\}$ such that

$$
E(u)=\inf \{E(v): v \in X \text { is a solution of problem }(1.1)\}
$$

An important application of the fractional Laplacian operator for dimension $N=1$ can be found in DipIERRO ET AL. [16]. We give a gist of the version of a model for the dynamics of the dislocation of atoms in crystals. The model is related to the Peierls-Nabarro energy functional. The system is a hybrid combination in which a discrete dislocation occurring 
along dislocation dynamics in crystals a slide line is incorporated in a continuum medium. The problem is as follows:

$$
\frac{\partial}{\partial t} v=(-\Delta)^{s} v-P^{\prime}(v)+\sigma_{\epsilon}(t, x), \quad \text { in }(0,+\infty) \times \mathbb{R},
$$

where $s \in\left[\frac{1}{2}, 1\right), P$ is a 1-periodic potential, and $\sigma_{\epsilon}$ plays the role of external stress acting on the material. Setting $v_{\epsilon}(t, x)=v\left(\frac{t}{\epsilon^{1+2 s}}, \frac{x}{\epsilon}\right)$, equation (2.18) can be recast as follows:

$$
\begin{aligned}
& \frac{\partial}{\partial t} v_{\epsilon}=\frac{1}{\epsilon}\left((-\Delta)^{s} v_{\epsilon}-\frac{1}{\epsilon^{2 s}} P^{\prime}\left(v_{\epsilon}\right)+\sigma_{\epsilon}(t, x)\right) \text { in }(0,+\infty) \times \mathbb{R}, \\
& v_{\epsilon}(0, x)=v_{\epsilon}^{0} \text { in } \mathbb{R} .
\end{aligned}
$$

For a suitable choice of $v_{\epsilon}^{0}$, the basic layer solution $u$ is introduced, which happens to be a solution to the following problem:

$$
\begin{aligned}
& (-\Delta)^{s} u-P^{\prime}(u)=0 \quad \text { in } \mathbb{R} \\
& u^{\prime}>0 \quad \text { and } \quad u(-\infty)=0, \quad u(0)=\frac{1}{2}, \quad u(+\infty)=1 .
\end{aligned}
$$

One can see that the problem considered in this article has a proper physical application and is a testimony to the importance of the problem considered in this paper.

\section{Auxiliary lemmas}

In this section we discuss some auxiliary lemmas and state the main result.

Lemma 3.1 (cf. Bö̈r AND Miyagaki [6, Lemma 2.1]) The space $X$ is continuously embedded in $H^{\frac{1}{2}}(\mathbb{R})$ and compactly embedded in $L^{p}(\mathbb{R})$ for every $p \geq 2$.

Below is the well-known Moser-Trudinger lemma (cf. CAO [9]).

Lemma 3.2 (cf. Ozawa [32]) Let $\left(u_{n}\right)$ be a sequence in $L^{2}(\mathbb{R})$ and $u \in L^{2}(\mathbb{R}) \backslash\{0\}$ such that $u_{n} \rightarrow$ u pointwise a.e. on $\mathbb{R}$. Moreover, let $\left(v_{n}\right)$ be a bounded sequence in $L^{2}(\mathbb{R})$ such that $\sup _{n \in \mathbb{N}}\left\{A\left(u_{n}^{2}, v_{n}^{2}\right)\right\}<\infty$. Then there exist $n_{0} \in \mathbb{N}$ and $C>0$ such that $\left\|u_{n}\right\|_{*}<C$ for any $n \geq n_{0}$. Moreover, if

$$
A\left(u_{n}^{2}, v_{n}^{2}\right) \rightarrow 0 \text { and }\left\|v_{n}\right\|_{2} \rightarrow 0 \quad \text { as } n \rightarrow \infty,
$$

then $\left\|v_{n}\right\|_{*} \rightarrow 0$ as $n \rightarrow \infty$.

Based on Lemma 2.1, Do Ó ET AL. [19] proved the following lemma.

Lemma 3.3 (cf. Do Ó ET AL. [19, Proposition 2.1]) For any $\beta>0, u \in W^{\frac{1}{2}}(\mathbb{R})$,

$$
\int_{\mathbb{R}}\left(e^{\beta u^{2}}-1\right) d x<\infty
$$


Hence, from Lemma 3.3 and equation (2.15), for any $u \in X$, we have

$$
\int_{\mathbb{R}}|F(u)| \leq \frac{\epsilon}{2}\|u\|^{2}+c_{2}\|u\|_{\rho_{2} q}^{q}\left(\int_{\mathbb{R}}\left(e^{\rho_{1} \beta u^{2}}-1\right) d x\right)^{\frac{1}{\rho_{1}}}<\infty .
$$

The following are some useful lemmas which will be used in the paper.

Lemma 3.4 (cf. Cingolani AND Weth [14, Lemma 2.6]) Let $\left(u_{n}\right),\left(v_{n}\right)$, and $\left(w_{n}\right)$ be bounded sequences in $X$ such that $u_{n} \rightarrow u$ in $X$. Then, for every $z \in X$, we have $A\left(v_{n} w_{n}, z\right.$. $\left.\left(u_{n}-u\right)\right) \rightarrow 0$ as $n \rightarrow \infty$.

Lemma 3.5 (cf. Felmer ET AL. [21, Lemma 2.2])

(i) The functionals $U, V, W$ are of class $C^{1}$ on $X$. In fact, $\left\langle U^{\prime}(u), v\right\rangle=4 A\left(u^{2}, u v\right)$, $\left\langle V^{\prime}(u), v\right\rangle=4 B\left(u^{2}, u v\right),\left\langle W^{\prime}(u), v\right\rangle=4 C\left(u^{2}, u v\right)$ for all $u, v \in X$.

(ii) $V$ is continuously differentiable on $L^{4}(\mathbb{R})$.

(iii) $U$ is a weakly lower semicontinuous functional on $H^{\frac{1}{2}}(\mathbb{R})$.

(iv) $E$ is lower semicontinuous on $H^{\frac{1}{2}}(\mathbb{R})$.

We now check that the energy functional $E$ depicts the mountain pass geometry. This will be required to obtain a Cerami sequence for a certain mountain pass energy level $d$ (given in Theorem 2.4). Following is the definition of a Cerami sequence pertaining to a $C^{1}$-functional.

Definition 3.6 (Soni And Choudhuri [39, Definition 2.1]) Let $\Phi: Y \rightarrow \mathbb{R}$ be a $C^{1}$ functional, where $Y$ is a normed linear space with the norm $\|\cdot\|_{Y}$. Then $\Phi$ is said to satisfy the Cerami condition at a level $c \in \mathbb{R}$ if any sequence $\left(u_{n}\right) \subset Y$ such that $\Phi\left(u_{n}\right) \rightarrow c$ and $\left(1+\left\|u_{n}\right\|_{Y}\right) \Phi^{\prime}\left(u_{n}\right) \rightarrow 0$ as $n \rightarrow \infty$ has a convergent subsequence in $Y$.

Remark 3.7 The advantage of this condition is that a Cerami sequence can produce a critical point even when a Palais-Smale sequence does not (cf. SCHECHTER [38]).

Lemma 3.8 There exist sufficiently small $R>0$ and $\mu_{0}>0$ such that

$$
m_{r}=\inf \{E(u): u \in X,\|u\|=r\}>0 \quad \text { for every } r \in(0, R]
$$

and

$$
m_{r}^{\prime}=\inf \left\{\left\langle E^{\prime}(u), u\right\rangle: u \in X,\|u\|=r\right\}>0 \quad \text { for every } r \in(0, R]
$$

whenever $\mu \in\left(0, \mu_{0}\right)$.

Proof Let $u \in X \backslash\{0\}$ and such that $u>\underline{u}_{\lambda}$ a.e. in $\Omega$. Choose $\rho_{1}, \rho_{2}>1$ such that $\rho_{1} \sim 1$, $\rho_{2}>2$, and $\rho_{1} \beta\|u\|^{2}$ in order to apply the exponential estimates in (2.17). Furthermore, on 
using the Sobolev embeddings, we get

$$
\begin{aligned}
E(u) & =\frac{1}{2}\|u\|^{2}+\frac{1}{4} W(u)-\int_{\mathbb{R}} F(u) d x-\frac{\mu}{1-\gamma} \int_{\mathbb{R}}|u|^{1-\gamma} d x \\
& \geq \frac{1}{2}\|u\|^{2}\left((1-\epsilon)-C_{2}\|u\|^{2}-C_{3}\|u\|^{q-2}-\frac{C_{4} \mu}{1-\gamma}\right) .
\end{aligned}
$$

Thus, for a pair of sufficiently small positive numbers $\left(\mu_{0}, R\right)$, we get $E(u)>0$, whenever $\|u\|=r<R$ and $\mu \in\left(0, \mu_{0}\right)$. Similarly,

$$
\begin{aligned}
\left\langle E^{\prime}(u), u\right\rangle & =\|u\|^{2}+W(u)-\int_{\mathbb{R}} f(u) u d x-\mu \int_{\mathbb{R}}|u|^{1-\gamma} d x \\
& \geq\|u\|^{2}\left((1-\epsilon)-C_{4}\|u\|^{2}-C_{5}\|u\|^{q-2}-C_{4} \mu\right) .
\end{aligned}
$$

Once again, by choosing $R>0$ and $\mu_{0}$ sufficiently small, we obtain $m_{r}^{\prime}>0$. This completes the proof.

Lemma 3.9 Let $u \in X \backslash\{0\}$ and $q>4$. Then

$$
\lim _{t \rightarrow 0} E(t u)=0, \quad \sup _{t>0}\{E(t u)\}<\infty, \quad E(t u) \rightarrow-\infty \quad \text { as } t \rightarrow \infty .
$$

Proof Suppose that $u \in X \backslash\{0\}$. By $\left(A_{4}\right)$ and $q>4$ we get

$$
\begin{aligned}
E(t u)= & \frac{t^{2}}{2}\|u\|^{2}+\frac{t^{4}}{4} W(u)-\int_{\mathbb{R}} F(t u) d x-\frac{t^{1-\gamma} \mu}{1-\gamma} \int_{\mathbb{R}}|u|^{1-\gamma} d x \\
\leq & \frac{t^{2}}{2}\|u\|^{2}+\frac{t^{4}}{4} W(u)-C_{q} t^{q} \int_{\mathbb{R}}|u|^{q} d x-\frac{t^{1-\gamma} \mu}{1-\gamma} \int_{\mathbb{R}}|u|^{1-\gamma} d x \\
\leq & \frac{t^{2}}{2}\|u\|^{2}+\frac{t^{4}}{4} W(u)-C_{q} t^{q}\|u\|_{q}^{q} \\
& -\frac{t^{1-\gamma} \mu}{1-\gamma} \int_{\mathbb{R}}|u|^{1-\gamma} d x \rightarrow-\infty \text { as } t \rightarrow \infty .
\end{aligned}
$$

Also, it is easy to see that $\lim _{t \rightarrow 0} E(t u)=0, \sup _{t>0}\{E(t u)\}<\infty$.

Remark 3.10 It is easy to verify invoking Lemma 3.9 and the intermediate value theorem that $0<m_{R} \leq d<\infty$. Since the functional $E$ has mountain pass geometry, by Du AND WETH [20, Lemma 3.2], there exists a sequence $\left(u_{n}\right) \subset X$ such that

$$
E\left(u_{n}\right) \rightarrow d \quad \text { and } \quad\left\|E^{\prime}\left(u_{n}\right)\right\|_{X^{\prime}}\left(1+\left\|u_{n}\right\|_{X}\right) \rightarrow 0 \quad \text { as } n \rightarrow \infty
$$

Lemma 3.11 Suppose that the sequence $\left(u_{n}\right) \subset X$ satisfies the Cerami condition (3.5). Then $\left(u_{n}\right)$ is bounded in $H^{\frac{1}{2}}(\mathbb{R})$. 
Proof Using (2.14), the condition in (3.5), $\left(A_{3}\right)$, and the embedding of $H^{\frac{1}{2}}(\mathbb{R}) \hookrightarrow L^{q}(\mathbb{R})$ for $q \in[1, \infty)$ from Lemma 3.1, we obtain

$$
\begin{aligned}
d+o(1) & \geq E\left(u_{n}\right)-\frac{1}{4}\left\langle E^{\prime}\left(u_{n}\right), u_{n}\right\rangle \\
& \geq \frac{1}{4}\left\|u_{n}\right\|^{2}+\left(\frac{L}{4}-1\right) \int_{\mathbb{R}} F\left(u_{n}\right) d x-\mu \frac{3+\gamma}{4(1-\gamma)} \int_{\mathbb{R}}|u|^{1-\gamma} d x \\
& \geq \frac{1}{4}\left\|u_{n}\right\|^{2}+C_{6}\left(\frac{L}{4}-1\right)\left\|u_{n}\right\|^{q}-\mu \frac{3+\gamma}{4(1-\gamma)} \int_{\mathbb{R}}|u|^{1-\gamma} d x \\
& \geq \frac{1}{4}\left\|u_{n}\right\|^{2}+C_{6}\left(\frac{L}{4}-1\right)\left\|u_{n}\right\|^{q}-C^{\prime} \mu \frac{3+\gamma}{4(1-\gamma)}\left\|u_{n}\right\|^{2} .
\end{aligned}
$$

The inequality in (3.6) clearly shows that the sequence $\left(u_{n}\right)$ is bounded in $H^{\frac{1}{2}}(\mathbb{R})$. For if not, then on dividing (3.6) by $\left\|u_{n}\right\|^{q}$ and then passing the limit $n \rightarrow \infty$ yields a contradiction to $0 \geq C_{6}\left(\frac{L}{4}-1\right)$. Thus, for a small range of $\mu$, say $\left(0, \mu_{0}\right)$, we have

$$
d+o(1) \geq \frac{1}{4}\left\|u_{n}\right\|^{2}
$$

The following lemma shows that any sequence $\left(u_{n}\right) \subset X$ such that $E\left(u_{n}\right) \leq d$ for all $n \in \mathbb{N}$ can be taken to be of sufficiently small norms.

Lemma 3.12 Let $\left(u_{n}\right) \subset X$ satisfy the Cerami condition in (3.5) with $q>4$. Then, for some sufficiently small $r_{0}>0$, we have lim sup $\left\|u_{n}\right\|^{2}<r_{0}^{2}$.

Proof By Lemma 3.11, we know that $\left(u_{n}\right)$ is bounded in $H^{\frac{1}{2}}(\mathbb{R})$. Certainly, lim sup $\left\|u_{n}\right\|^{2} \leq$ $4 c+o(1)$ is bounded above (and of course, below). We will find an estimate of the upper bound for this quantity. Consider the set $\mathfrak{S}=\{u \in X: u \neq 0, W(u) \leq 0\}$ and define $u_{t}(x)=$ $t^{2} u(t x)$ for all $t>0, u \neq 0 \in X, x \in \mathbb{R}$. We have $W\left(u_{t}\right)=t^{6} C(U)-t^{6} \ln t\|u\|_{2}^{4} \rightarrow-\infty$ as $t \rightarrow \infty$. This shows that $\mathfrak{S}$ is nonempty. By the Sobolev embedding theorem we have $\|u\| \geq C\|u\|_{q}$ for all $u \in H^{\frac{1}{2}}(\mathbb{R}) \backslash\{0\}$.

We define

$$
S_{q}(v)=\frac{\|v\|}{\|v\|_{q}} .
$$

Therefore, $S_{q}=\inf _{v \in \mathfrak{S}} S_{q}(v) \geq \inf _{v \neq 0} S_{q}(v)>0$. We will now estimate the energy level $d$. Let $v \in \mathfrak{S}$ and $T>0$ be sufficiently small. Then $E(T v)<0$. Take a path $\alpha:[0,1] \rightarrow X$ defined as $\alpha(t)=t T v$. Therefore,

$$
d \leq \max _{0 \leq t \leq 1} E(\alpha(t))=\max _{0 \leq t \leq 1} E(t T v) \leq \max _{t \geq 0} E(t v)
$$

Consequently, for $w \in \mathfrak{S}$, we have

$$
d \leq \max _{t \geq 0} E(t w) \leq \max _{t \geq 0}\left\{\frac{t^{2}}{2}\|w\|^{2}-C_{q} t^{q}\|w\|_{q}^{q}\right\} \leq\left(\frac{1}{2}-\frac{1}{q}\right) \frac{\left(S_{q}(w)\right)^{\frac{2 q}{q-2}}}{\left(q C_{q}\right)^{\frac{2}{q-2}}}
$$


where we have used $\left(A_{4}\right)$. On taking infimum over $w \in \mathfrak{S}$, we obtain

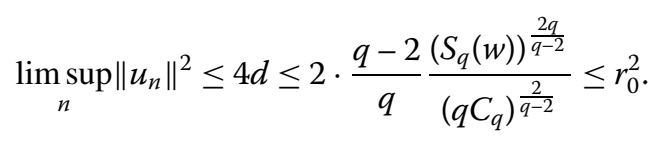

Before we state and prove the next lemma, we need to recall the following two theorems.

Theorem 3.13 (Di Nezza ET AL. [15, Theorem 7.1]) Let $s \in(0,1), p \in[1, \infty), q \in[1, p]$, $\Omega \subset \mathbb{R}^{N}$ be a bounded extension domain for $W^{s, p}$ and $T$ be a bounded subset of $L^{p}(\Omega)$. Suppose that

$$
\sup _{f \in T} \iint_{\Omega \times \Omega} \frac{|f(x)-f(y)|^{p}}{|x-y|^{N+s p}} d x d y<\infty
$$

Then $T$ is pre-compact in $L^{q}(\Omega)$.

Theorem 3.14 (Adams [2, Theorem 7.41]) Suppose that for $\Omega \subset \mathbb{R}^{N}$ there exists a strong $(M+1)$-extension operator $\mathfrak{O}$ and, for $|\delta| \leq|\alpha|=M$, linear operators $\mathfrak{O}_{\alpha \delta}$ continuous from $W^{s, p}(\Omega)$ into $W^{1, p}\left(\mathbb{R}^{N}\right)$ and from $L^{p}(\Omega)$ into $L^{p}\left(\mathbb{R}^{N}\right)$ such that if $u \in W^{m, p}(\Omega)$, then

$$
D^{\alpha} \mathfrak{O} u(x)=\sum_{|\delta| \leq M} \mathfrak{D}_{\alpha \delta} D^{\delta} u(x)
$$

If $s=M+\sigma>0,0 \leq \sigma<1$, then $W^{s, p}(\Omega)$ coincides with the set of restrictions on $\Omega$ of functions in $W^{s, p}\left(\mathbb{R}^{N}\right)$.

Finally, we have the following lemma.

Lemma 3.15 Let $\left(u_{n}\right) \subset X$ be bounded in $H^{\frac{1}{2}}(\mathbb{R})$ and such that

$$
Q=\operatorname{liminfsup}_{y \in \mathbb{Z}} \int_{B_{\frac{3}{2}}(y)}\left|u_{n}(x)\right|^{2} d x>0 .
$$

Then there exist $u \in H^{\frac{1}{2}}(\mathbb{R}) \backslash\{0\}$ and $\left(y_{n}\right) \subset \mathbb{Z}$ such that, up to a subsequence, $\tilde{u}_{n}=u_{n}(\cdot-$ $\left.y_{n}\right) \rightarrow u \in H^{\frac{1}{2}}(\mathbb{R})$. Here, $B_{\frac{3}{2}}(y)=\left\{x \in \mathbb{R}:|x-y|<\frac{3}{2}\right\}$.

Proof The property of liminf and sup together produces the sequence $\left(y_{n}\right)$ such that $\left|y_{n}\right| \rightarrow$ $\infty$ and the boundedness of $\left(u_{n}\right)$ in $H^{\frac{1}{2}}(\mathbb{R})$ produces $u$ such that $u_{n} \rightarrow u \in H^{\frac{1}{2}}(\mathbb{R})$. Also, $u \neq 0$ given condition (3.12). By Lemma 3.1 we have that $u_{n} \rightarrow u$ in $L^{2}(\mathbb{R})$ and hence from Theorem 3.13 we have $u_{n} \rightarrow u$ in $L^{2}\left(B_{\frac{3}{2}}\left(y_{n}\right)\right)$. Therefore, there exists a subsequence such that $u_{n} \rightarrow u$ a.e. in $B_{\frac{3}{2}}\left(y_{n}\right)$. These $\tilde{u}_{n}=u_{n}\left(\cdot-y_{n}\right)$ are nothing but the restrictions of the functions in $\left(u_{n}\right)$ over $B_{\frac{3}{2}}\left(y_{n}\right)$. Thus by Theorem 3.14, we can consider the functions $\mathfrak{O}_{00} \tilde{u}_{n}$ that will still be denoted by $\tilde{u}_{n}$. Therefore, $\tilde{u}_{n} \rightarrow u \in H^{\frac{1}{2}}(\mathbb{R})$. 


\section{Proof of the main theorem}

We now give a proof of Theorem 2.4. First, we will prove another lemma.

Lemma 4.1 Let $q>2$ and $\left(u_{n}\right) \subset X$ satisfying the Cerami condition. On passing to a subsequence, if necessary, exactly one of the following statements holds true.

(i) $\left\|u_{n}\right\| \rightarrow 0$ and $E\left(u_{n}\right) \rightarrow 0$ as $n \rightarrow \infty$.

(ii) There exists $\left(y_{n}\right) \subset \mathbb{Z}$ such that $\left|y_{n}\right| \rightarrow \infty$ such that $\tilde{u}_{n}=u_{n}\left(\cdot-y_{n}\right) \rightarrow u$ in $X$ for $a$ nontrivial critical point $u \in X$ of $E$.

Proof By Lemma 3.12, we see that the Cerami sequence $\left(u_{n}\right)$ is bounded in $H^{\frac{1}{2}}(\mathbb{R})$. Therefore there exists a subsequence from Lemma 3.15 such that

$$
\int_{\mathbb{R}}\left(e^{\rho_{1} \beta u_{n}^{2}}-1\right) d x \leq C_{\beta} \quad \text { for every } n \in \mathbb{N} \text {. }
$$

Suppose that (i) does not hold.

Claim A $\operatorname{liminfsup}_{n} \int_{B_{2}(y)}\left|u_{n}(x)\right|^{2} d x>0$.

On the contrary, suppose this does not hold. By Lion's Lemma 2.4 in YU ET AL. [42], we have $u_{n} \rightarrow 0$ in $L^{a}(\mathbb{R})$ for all $a>2$. By (2.8), we have $V\left(u_{n}\right) \rightarrow 0$ as $n \rightarrow \infty$. Since $q>2$, we have by Remark 2.2 for a subsequence that

$$
\begin{aligned}
& \left|\int_{\mathbb{R}} f\left(u_{n}\right) u_{n} d x\right| \leq \epsilon\left\|u_{n}\right\|_{2}^{2}+C_{7}\left\|u_{n}\right\|_{q r_{2}}^{q} \rightarrow 0, \\
& \int_{\mathbb{R}}\left|u_{n}\right|^{1-\gamma} d x \rightarrow 0 \quad \text { as } \epsilon \rightarrow 0, n \rightarrow \infty
\end{aligned}
$$

Furthermore,

$$
\begin{aligned}
\left\|u_{n}\right\|^{2}+U\left(u_{n}\right)= & \left\langle E^{\prime}\left(u_{n}\right), u_{n}\right\rangle+V\left(u_{n}\right)+\int_{\mathbb{R}} f\left(u_{n}\right) u_{n} d x \\
& +\mu \int_{\mathbb{R}}\left|u_{n}\right|^{1-\gamma} d x \rightarrow 0 \text { as } n \rightarrow \infty
\end{aligned}
$$

This further implies that $\left\|u_{n}\right\|, U\left(u_{n}\right) \rightarrow 0$ as $n \rightarrow \infty$. By the embedding $H^{\frac{1}{2}}(\mathbb{R}) \hookrightarrow L^{2}(\mathbb{R})$, we have that $\left\|u_{n}\right\|_{2} \rightarrow 0$ as $n \rightarrow \infty$. Also, by Remark 2.2, we have $\int_{\mathbb{R}} F\left(u_{n}\right) d x \rightarrow 0$ as $n \rightarrow$ $\infty$. Thus $E\left(u_{n}\right) \rightarrow 0$ as $n \rightarrow \infty$, which is a contradiction. Thus

$$
\operatorname{liminfsup}_{n} \int_{y \in \mathbb{Z}}\left|u_{B_{2}(y)}(x)\right|^{2} d x>0 .
$$

This fact combined with Lemma 3.15 helps us to produce a sequence $\left(y_{n}\right) \subset \mathbb{Z}$ and $u \in$ $H^{\frac{1}{2}}(\mathbb{R}) \backslash\{0\}$ such that $u_{n}\left(\cdot-y_{n}\right)=\tilde{u}_{n} \rightarrow u$ in $H^{\frac{1}{2}}(\mathbb{R})$. Therefore $\left(\tilde{u}_{n}\right)$ is bounded in $L^{p}(\mathbb{R})$ for any $p \geq 2$ and hence $\tilde{u}_{n}(x) \rightarrow u(x)$ a.e. in $\mathbb{R}$. Now, observe that for $q>2$

$$
\begin{aligned}
U\left(\tilde{u}_{n}\right)=U\left(u_{n}\right) & =\left\langle E^{\prime}\left(u_{n}\right), u_{n}\right\rangle+V\left(u_{n}\right)+\int_{\mathbb{R}} f\left(u_{n}\right) u_{n} d x+\mu \int_{\mathbb{R}}\left|u_{n}\right|^{1-\gamma} d x-\left\|u_{n}\right\|^{2} \\
& \leq C_{0}\left\|u_{n}\right\|_{4}^{2}+\epsilon C_{8}+C_{9}\left\|u_{n}\right\|_{q r_{2}}^{q}+o(1) \leq C_{10}+o(1) .
\end{aligned}
$$


Therefore, $U\left(\tilde{u}_{n}\right)<\infty$. Hence, since $\left(\tilde{u}_{n}\right)$ is bounded in $L^{2}(\mathbb{R})$, it follows by Lemma 3.2 that $\left(\left\|\tilde{u}_{n}\right\|_{*}\right)$ is bounded in $X$. By the reflexivity of $X$, we get $\tilde{u}_{n} \rightarrow u$ in $X$. From Lemma 3.1 we obtain $\tilde{u}_{n} \rightarrow u$ in $L^{p}(\mathbb{R})$ for all $p \geq 2$.

Claim B $\left\langle E^{\prime}\left(\tilde{u}_{n}\right), \tilde{u}_{n}-u\right\rangle \rightarrow 0$ as $n \rightarrow \infty$.

A simple change of variable guarantees that $\left\langle E^{\prime}\left(\tilde{u}_{n}\right), \tilde{u}_{n}-u\right\rangle=\left\langle E^{\prime}\left(u_{n}\right), u_{n}-u\left(x+y_{n}\right)\right\rangle$. Thus,

$$
\begin{aligned}
\left|\left\langle E^{\prime}\left(\tilde{u}_{n}\right), \tilde{u}_{n}-u\right\rangle\right| & =\left|\left\langle E^{\prime}\left(u_{n}\right), u_{n}-u\left(x+y_{n}\right)\right\rangle\right| \\
& \leq\left\|E^{\prime}\left(u_{n}\right)\right\|_{X^{\prime}}\left(\left\|u_{n}\right\|_{X}+\left\|u\left(\cdot+y_{n}\right)\right\|_{X}\right) \quad \text { for every } n \in \mathbb{N} .
\end{aligned}
$$

We will now try to estimate $\left\|u\left(\cdot+y_{n}\right)\right\|_{X}$. Suppose that $\left|y_{n}\right| \rightarrow \infty$. Then, for each $x \in \mathbb{R}$,

$$
\ln \left(1+\left|y_{n}\right|\right)-\ln \left(1+\left|x-y_{n}\right|\right)=\ln \left(\frac{1+\left|y_{n}\right|}{1+\left|x-y_{n}\right|}\right) \rightarrow 0, \quad n \rightarrow \infty
$$

Thus there exists $C_{11}>0$ such that $\ln \left(1+\left|x-y_{n}\right|\right) \geq C_{11} \ln \left(1+\left|y_{n}\right|\right)$ for all $n \in \mathbb{N}$, which implies

$$
\begin{aligned}
\left\|u_{n}\right\|_{*}^{2} & \geq \int_{\mathbb{R}} \ln \left(1+\left|x-y_{n}\right|\right) \tilde{u}_{n}^{2} d x \\
& \geq C_{11} \ln \left(1+\left|y_{n}\right|\right) \int_{\mathbb{R}} \tilde{u}_{n}^{2} d x \geq C_{11} \ln \left(1+\left|y_{n}\right|\right) \quad \text { for every } n \in \mathbb{N} .
\end{aligned}
$$

Now, if $y_{n} \rightarrow y_{0}$, then obviously up to a subsequence still denoted by $\left(y_{n}\right)$ we have $y_{n}=y_{0}$. Let $y_{0}>0$. Suppose that $\delta>0$ and define $S=(-b, 0)$. For each $x \in S$, we have $\left|x-y_{0}\right|>\left|y_{0}\right|$. Thus, by the mean value theorem of integration, there exists $x_{b} \in S$ such that

$$
\begin{aligned}
\left\|\tilde{u}_{n}\right\|_{*}^{2} & \geq \int_{S} \ln \left(1+\left|x-y_{n}\right|\right) \tilde{u}_{n}^{2} d x \\
& =a \ln \left(1+\left|x-y_{n}\right|\right) \tilde{u}_{n}^{2}\left(x_{a}\right) \\
& =C_{11}^{\prime} \ln \left(1+\left|x-y_{n}\right|\right) \geq C_{11}^{\prime} \ln \left(1+\left|y_{0}\right|\right) \\
& =C_{11}^{\prime} \ln \left(1+\left|y_{n}\right|\right) \quad \text { for some } C_{11}^{\prime}>0 .
\end{aligned}
$$

Similarly, for the case of $y_{0}<0$, we arrive at a similar conclusion. Furthermore, when $y_{0}=0$, we have $\tilde{u}_{n}=u_{n}$, and hence the conclusion is straightforward. Thus, for any of the cases, there exists $C_{11}^{\prime}>0$ (need not be the same constant but is denoted by the same name) such that

$$
\left\|u_{n}\right\|_{*}^{2} \geq C_{11}^{\prime} \ln \left(1+\left|y_{n}\right|\right) \quad \text { for every } n \in \mathbb{N} \text {. }
$$

From (2.9) we obtain

$$
\left\|\tilde{u}_{n}\right\|_{*}^{2}=\int_{\mathbb{R}} \ln \left(1+\left|x+y_{n}\right|\right) u_{n}^{2} d x \leq\left\|u_{n}\right\|_{*}^{2}+\ln \left(1+\left|y_{n}\right|\right)\left\|u_{n}\right\|_{2}^{2}
$$


By the weak lower semicontinuity of norm and by the $\mathbb{Z}$-invariance of $\|\cdot\|_{2}$, it follows from (2.9), (4.8), (4.10) that

$$
\begin{aligned}
\left\|u\left(\cdot+y_{n}\right)\right\|_{*}^{2} & \leq\|u\|_{*}^{2}+\ln \left(1+\left|y_{n}\right|\right)\|u\|_{2}^{2} \\
& \leq\left\|\tilde{u}_{n}\right\|_{*}^{2}+\ln \left(1+\left|y_{n}\right|\right)\left\|u_{n}\right\|_{2}^{2} \\
& =\left\|u_{n}\right\|^{2}+\left\|u_{n}\right\|_{*}^{2}\left(1+C_{12}\left\|u_{n}\right\|_{2}^{2}\right) \\
& \leq\left\|u_{n}\right\|^{2}+C_{13}\left\|u_{n}\right\|_{*}^{2} \leq C_{14}\left\|u_{n}\right\|_{X}^{2}
\end{aligned}
$$

for $n \in \mathbb{N}$. This implies that there exists $C_{15}>0$ such that, on passing to a subsequence, we obtain

$$
\left\|u\left(\cdot+y_{n}\right)\right\|_{*}^{2}=\|u\|^{2}+\left\|u\left(\cdot+y_{n}\right)\right\|_{*}^{2} \leq\left\|u_{n}\right\|^{2}+C_{14}\left\|u_{n}\right\|_{X}^{2} \leq C_{15}\left\|u_{n}\right\|_{X}^{2} .
$$

By the Cerami condition, we have

$$
\left|\left\langle E^{\prime}\left(\tilde{u}_{n}\right), \tilde{u}_{n}-u\right\rangle\right| \leq\left(1+\sqrt{C_{15}}\right)\left\|E^{\prime}\left(u_{n}\right)\right\|_{X^{\prime}}\left\|u_{n}\right\|_{X} \rightarrow 0, \quad \text { as } n \rightarrow \infty .
$$

Claim C $\int_{\mathbb{R}} f\left(\tilde{u}_{n}\right)\left(\tilde{u}_{n}-u\right) d x \rightarrow 0$, as $n \rightarrow \infty$.

By the $\mathbb{Z}$-invariance of $\|\cdot\|$, the Moser-Trudinger inequality, and (2.17), we get

$$
\left|\int_{\mathbb{R}} f\left(\tilde{u}_{n}\right)\left(\tilde{u}_{n}-u\right) d x\right| \leq\left\|\tilde{u}_{n}\right\|_{2}\left\|\tilde{u}_{n}-u\right\|_{2}+C_{16}\left\|\tilde{u}_{n}-u\right\|_{q r_{2}}^{q} \rightarrow 0, \quad \text { as } n \rightarrow \infty .
$$

Hence Claim $C$ has been verified.

Furthermore,

$$
\mid\left\langle V^{\prime}\left(\tilde{u}_{n}\right), \tilde{u}_{n}-u\left\|\mid \leq c_{0}\right\| \tilde{u}_{n}\left\|_{4}^{3}\right\| \tilde{u}_{n}-u \|_{4} \rightarrow 0\right.
$$

and

$$
\left\langle U^{\prime}\left(\tilde{u}_{n}\right), \tilde{u}_{n}-u\right\rangle=A\left(\tilde{u}_{n}^{2}, \tilde{u}_{n}\left(\tilde{u}_{n}-u\right)^{2}\right)=A\left(\tilde{u}_{n}^{2},\left(\tilde{u}_{n}-u\right)^{2}\right)+A\left(\tilde{u}_{n}^{2}, u\left(\tilde{u}_{n}-u\right)^{2}\right) .
$$

Since $\left(\tilde{u}_{n}\right)$ is bounded in $X$, invoking Lemma 3.4, we obtain $A\left(\tilde{u}_{n}^{2}, u\left(\tilde{u}_{n}-u\right)^{2}\right) \rightarrow 0$, as $n \rightarrow$ $\infty$. Thus

$$
o(1)=\left\|\tilde{u}_{n}\right\|^{2}-\|u\|^{2}+A\left(\tilde{u}_{n}^{2},\left(\tilde{u}_{n}-u\right)^{2}\right)+o(1) \geq\left\|\tilde{u}_{n}\right\|^{2}-\|u\|^{2}+o(1) .
$$

Hence $\left\|\tilde{u}_{n}\right\| \rightarrow\|u\|$ and $A\left(\tilde{u}_{n}^{2},\left(\tilde{u}_{n}-u\right)^{2}\right) \rightarrow 0$. Therefore $\left\|\tilde{u}_{n}-u\right\| \rightarrow 0$ as $n \rightarrow \infty$. Furthermore, by Lemma 3.2, we have $\left\|\tilde{u}_{n}-u\right\|_{*} \rightarrow 0$ which implies that $\tilde{u}_{n} \rightarrow u$ in $X$ as $n \rightarrow \infty$. It remains to show that $u$ is indeed a critical point of the functional $E$. As deduced in (4.11), one can show that there exists $C_{17}>0$ such that

$$
\left\|v\left(\cdot+y_{n}\right)\right\|_{X} \leq C_{17}\left\|u_{n}\right\|_{X} \quad \text { for every } n \in \mathbb{N}
$$


Therefore,

$$
\begin{aligned}
\left|\left\langle E^{\prime}(u), v\right\rangle\right| & =\lim _{n \rightarrow \infty}\left|\left\langle E^{\prime}\left(\tilde{u}_{n}\right), v\right\rangle\right|=\lim _{n \rightarrow \infty}\left|\left\langle E^{\prime}\left(u_{n}\right), v\left(\cdot+y_{n}\right)\right\rangle\right| \\
& =C_{17} \lim _{n \rightarrow \infty}\left\|E^{\prime}\left(u_{n}\right)\right\|_{X^{\prime}}\left\|u_{n}\right\|_{X} \\
& =0 \quad \text { for every } v \in X .
\end{aligned}
$$

\section{Proof of Theorem 2.4:}

(i) By Lemma 3.8, Remark 3.10, and Lemma 4.1, we can conclude that there exists $u_{0}$, a critical point of $E$, such that $E\left(u_{0}\right)=d$.

(ii) Define

$$
D=\left\{v \in X \backslash\{0\}: E^{\prime}(v)=0\right\} .
$$

Clearly, $D \neq \emptyset$ since $E^{\prime}\left(u_{0}\right)=0$. This allows us to consider $\left(u_{n}\right) \subset D$ satisfying $E\left(u_{n}\right) \rightarrow d^{\prime}=\inf _{v \in D}\{E(v)\}$. Clearly, $d^{\prime} \in[-\infty, d]$ by the definition of $d^{\prime}$. Obviously, if $d=d^{\prime}$, then we already have a solution, namely $u_{0}$. Also, if $d^{\prime}=0$, then we choose the sequence $\left(u_{n}\right)$ such that $E\left(u_{n}\right) \rightarrow 0$ as $n \rightarrow \infty$ but $\left\|u_{n}\right\| \nrightarrow 0$ since (a) 0 is not a critical point of $E$, (b) this choice of the sequence $\left(u_{n}\right)$ helps to rule out (i) of Lemma 4.1.

Otherwise, if $0 \neq d^{\prime}<d$, there exists a subsequence, still denoted by $\left(u_{n}\right)$, such that $E\left(u_{n}\right)<d$ for all $n$. Thus, Lemma 3.12 holds for this subsequence as well and the results thus derived as a consequence of Lemma 3.12 are all applicable.

Furthermore, by using Lemma 3.8 and Lemma 4.1, there exists $\left(y_{n}\right) \subset \mathbb{Z}$ such that $\tilde{u}_{n} \rightarrow u$ in $X$, where $u$ turned out to be a nontrivial critical point of $E$ in $X$ by (ii) of Lemma 4.1. Hence, $E^{\prime}(u)=\lim E^{\prime}\left(u_{n}\right)=0$. This implies that $u \in D$ and $E(u)=\lim _{n} E\left(\tilde{u}_{n}\right)=\lim _{n} E\left(u_{n}\right)=d^{\prime}>-\infty$.

\section{Appendix}

Lemma A.2 will establish the existence of a positive solution to (2.13) and Lemma A.4 will guarantee that a solution to (1.1) is greater than or equal to the solution to (2.13).

Lemma A.1 (Weak comparison principle) Let $u, v \in X$. Suppose that $-\Delta^{\frac{1}{2}} v-\frac{\mu}{v^{\gamma}} \geq-\Delta^{\frac{1}{2}} u-$ $\frac{\mu}{u^{\gamma}}$ weakly in $\mathbb{R}$. Then $v \geq u$ in $\mathbb{R}$.

Proof The idea was motivated by SAOUdI ET AL. [37]. Since $-\Delta^{\frac{1}{2}} v-\frac{\mu}{v^{\gamma}} \geq-\Delta^{\frac{1}{2}} u-\frac{\mu}{u^{\gamma}}$ weakly in $\mathbb{R}$, we have

$$
\left\langle-\Delta^{\frac{1}{2}} v, \phi\right\rangle-\int_{\mathbb{R}} \frac{\mu \phi}{v^{\gamma}} d x \geq\left\langle-\Delta^{\frac{1}{2}} u, \phi\right\rangle-\int_{\mathbb{R}} \frac{\mu \phi}{u^{\gamma}} d x
$$

for every $\phi \geq 0 \in X$.

In particular, choose $\phi=(u-v)^{+}$. Then the inequality in (A.1) looks as follows:

$$
\left\langle-\Delta^{\frac{1}{2}} v+\Delta^{\frac{1}{2}} u,(u-v)^{+}\right\rangle-\mu \int_{\mathbb{R}_{+}}(u-v)^{+}\left(\frac{1}{v^{\gamma}}-\frac{1}{u^{\gamma}}\right) d x \geq 0,
$$


where $\mathbb{R}_{+}=\{x: u(x)>v(x)\}$. Let $\psi=u-v$. We choose the test function $\phi=(u-v)^{+}$. We express

$$
\psi=u-v=(u-v)^{+}-(u-v)^{-}
$$

to obtain

$$
[\psi(y)-\psi(x)][\phi(x)-\phi(y)]=-\left(\psi^{+}(x)-\psi^{+}(y)\right)^{2} .
$$

The equation in (A.3) implies

$$
0 \geq\left\langle-\Delta^{\frac{1}{2}} v+\Delta^{\frac{1}{2}} u,(v-u)^{+}\right\rangle=-\int_{\mathbb{R}} \frac{1}{|x-y|^{2}}\left(\psi^{+}(x)-\psi^{+}(y)\right)^{2} d x d y \geq 0 .
$$

This leads to the conclusion about the Lebesgue measure of $\mathbb{R}_{+}$, i.e., $\left|\mathbb{R}_{+}\right|=0$. In other words $v \geq u$ a.e. in $\mathbb{R}$.

Lemma A.2 Let $\mu>0$. Then the following problem

$$
\begin{aligned}
& (-\Delta)^{\frac{1}{2}} u+u+\left(\ln |\cdot| *|u|^{2}\right)=\mu u^{-\gamma} \quad \text { in } \mathbb{R}, \\
& u>0 \quad \text { in } \mathbb{R}
\end{aligned}
$$

has a unique weak solution in $X_{0}$. This solution is denoted by $\underline{u}_{\mu}$, satisfies $\underline{u}_{\mu} \geq \epsilon_{\mu} v_{0}$ a.e. in $\Omega$, where $\epsilon_{\mu}>0$ is a constant. Here $v_{0}>0$ is a suitable function such that $E\left(\epsilon_{\mu} v_{0}\right)<0$.

Proof We follow the proof in Choudhuri [11] and Choudhuri And SaOudi [12]. First, we note that an energy functional on $X$ formally corresponding to (A.5) can be defined as follows:

$$
E(u)=\frac{1}{2}\|u\|^{2}+W(u)-\frac{\mu}{1-\gamma} \int_{\Omega}\left(u^{+}\right)^{1-\gamma} d x
$$

for all $u \in X$ where $u^{+}(x)=\max \{u(x), 0\}$. By using the Poincaré inequality, this functional is coercive and continuous on $X$. It follows that $E$ possesses a global minimizer $u_{0} \in X$. Obviously, $u_{0} \neq 0$ since $E(0)=0>E\left(\epsilon v_{0}\right)$ for sufficiently small $\epsilon$ and some $v_{0}>0$ in $\mathbb{R}$.

Next, if $u_{0}$ is a global minimizer for $E$, then $\left|u_{0}\right|$ is also a global minimizer. This is because $E\left(\left|u_{0}\right|\right) \leq E\left(u_{0}\right)$. Clearly, the equality holds if and only if $u_{0}^{-}=0$ a.e. in $\mathbb{R}$. Here $u^{-}(x)=$ $\min \{-u(x), 0\}$. In other words, we must have $u_{0} \geq 0$, i.e., $u_{0} \in X^{+}$, where

$$
X^{+}=\{u \in X: u \geq 0 \text { a.e. in } \mathbb{R}\}
$$

is the positive cone in $X$.

Furthermore, we will show that $u_{0} \geq \epsilon v_{0}>0$ holds a.e. in $\mathbb{R}$ for small enough $\epsilon$. We observe that

$$
\left.\frac{d}{d t} E\left(t v_{0}\right)\right|_{t=\epsilon}=\epsilon\left\|v_{0}\right\|^{2}+4 \epsilon^{3} C\left(v_{0}^{4}\right)-\mu \epsilon^{-\gamma} \int_{\Omega} v_{0}^{1-\gamma} d x<0
$$


whenever $\epsilon \in\left(0, \epsilon_{\mu}\right]$ for some sufficiently small $\epsilon_{\mu}$. We now show that $u_{0} \geq \epsilon_{\mu} \nu_{0}$. Suppose we assume the contrary that $w=\left(\epsilon_{\mu} v_{0}-u_{0}\right)^{+}$does not vanish identically in $\mathbb{R}$. We denote $\mathbb{R}_{+}=\{x \in \mathbb{R}: w(x)>0\}$. We will analyze the function $\zeta(t)=E\left(u_{0}+t w\right)$ of $t \geq 0$. This function is convex when defined over $X^{+}$being convex. Furthermore, $\zeta^{\prime}(t)=\left\langle E^{\prime}\left(u_{0}+t w\right), w\right\rangle$ is nonnegative and nondecreasing for $t>0$. Consequently, for $0<t<1$, we have

$$
0 \leq \zeta^{\prime}(1)-\zeta^{\prime}(t)=\left\langle E^{\prime}\left(u_{0}+w\right)-E^{\prime}\left(u_{0}+t w\right), w\right\rangle=\int_{\mathbb{R}_{+}} E^{\prime}\left(u_{0}+w\right) d x-\zeta^{\prime}(t)<0
$$

by inequality (A.7) and $\zeta^{\prime}(t) \geq 0$ with $\zeta^{\prime}(t)$ being nondecreasing for every $t>0$, which leads to a contradiction. Therefore $w=0$ in $\mathbb{R}$ and hence $u_{0} \geq \epsilon_{\mu} v_{0}$ a.e. in $\mathbb{R}$.

Moreover, since the functional $E$ is strictly convex on $X^{+}$, we conclude that $u_{0}$ is the only critical point of $E$ in $X^{+}$with the property ess $\inf u_{0}>0$ for any compact subset $V \subset \mathbb{R}$. Thus we choose $\underline{u}_{\mu}=u_{0}$ in the cutoff functional.

Remark A.3 We now perform an analysis on a solution (if it exists). Suppose that $u$ is a solution to (1.1). Then we observe the following:

1. If $u$ is a global minimizer, then clearly $E(u) \leq E(|u|)$. Further, $E(u) \geq E(|u|)$ is always true due to the first term of the energy functional. Thus $u^{-}=0$ a.e. in $\mathbb{R}$.

2. In fact, a solution to (1.1) can be considered to be positive, i.e., $u>0$ a.e. in $\mathbb{R}$, due to the presence of the singular term.

Therefore, without loss of generality, we may assume that the solution is positive.

We finally have the following result.

Lemma A.4 (A priori analysis) Fix $a \mu \in\left(0, \mu_{0}\right)$. Then a solution of (1.1), say $u>0$, is such that $u>\underline{u}_{\mu}$ a.e. in $\mathbb{R}$.

Proof Fix $\mu \in\left(0, \mu_{0}\right)$ and let $u \in X$ be a positive solution to (1.1) and $\underline{u}_{\mu}>0$ be a solution to (A.5). We will show that $u \geq \underline{u}_{\mu}$ a.e. in $\mathbb{R}$. Thus, we let $\mathbb{R}^{*}=\left\{x \in \mathbb{R}: u(x)<\underline{u}_{\mu}(x)\right\}$, and from the equation satisfied by $u, \underline{u}_{\mu}$ we have

$$
\begin{aligned}
0 \leq & \left\langle\Delta^{\frac{1}{2}}\left(\underline{u}_{\mu}-u\right), \underline{u}_{\mu}-u\right\rangle_{\mathbb{R}}+\left\langle W^{\prime}\left(\underline{u}_{\mu}\right)-W^{\prime}(u), \underline{u}_{\mu}-u\right\rangle \\
& +\int_{\mathbb{R}}\left(f(u)-f\left(\underline{u}_{\mu}\right)\right)\left(\underline{u}_{\mu}-u\right) d x \\
\leq & \mu \int_{\mathbb{R}^{*}}\left(\underline{u}_{\mu}^{-\gamma}-u^{-\gamma}\right)\left(\underline{u}_{\mu}-u\right) d x ; \quad \text { by }\left(A_{1}\right) \leq 0 .
\end{aligned}
$$

Furthermore, we have

$$
\left\langle\Delta^{\frac{1}{2}}\left(\underline{u}_{\mu}-u\right), \underline{u}_{\mu}-u\right\rangle_{\mathbb{R}^{*}} \geq 0
$$

Hence, by (A.9) and (A.10), we obtain $u \geq \underline{u}_{\mu}$ a.e. in $\mathbb{R}$.

Now suppose $S=\left\{x \in \Omega: u(x)=\underline{u}_{\lambda}(x)\right\}$. Clearly $S$ is a measurable set, and hence for any $\delta>0$ there exists a closed subset $F$ of $S$ such that $|S \backslash F|<\delta$. Furthermore, let $|S|>0$. Define 
a test function $\varphi \in C_{c}^{1}(\mathbb{R})$ such that

$$
\varphi(x)= \begin{cases}1, & \text { if } x \in F, \\ 0<\varphi<1, & \text { if } x \in S \backslash F, \\ 0, & \text { if } x \in \mathbb{R} \backslash S .\end{cases}
$$

Since $u$ is a weak solution to (1.1), we have

$$
\begin{aligned}
0= & \left(\left\langle(-\Delta)^{\frac{1}{2}} u, \varphi\right\rangle_{\mathbb{R}}+\int_{F} W^{\prime}(u) d x+\int_{S \backslash F} W^{\prime}(u) \varphi d x-\mu \int_{F} u^{-\gamma} d x\right. \\
& -\mu \int_{S \backslash F} u^{-\gamma} \varphi d x-\int_{F} f(u) d x-\int_{S \backslash F} f(u) \varphi d x \\
= & -\mu \int_{F} u^{-\gamma} d x-\mu \int_{S \backslash F} u^{-\gamma} \varphi d x-\int_{F} f(u) d x-\int_{S \backslash F} f(u) \varphi d x<0 .
\end{aligned}
$$

This is a contradiction. Therefore, $|S|=0$. Hence, $u>\underline{u}_{\lambda}$ a.e. in $\mathbb{R}$.

\section{Acknowledgements}

Not applicable.

\section{Funding}

The first author was supported by the Indian Council of Scientific and Industrial Research grant 25(0292)/18/EMR-II. The second author was supported by the Slovenian Research Agency grants P1-0292, N1-0114, N1-0083, N1-0064, and J1-8131.

Availability of data and materials

Not applicable.

\section{Declarations}

Competing interests

The authors declare that they have no competing interests.

\section{Authors' contributions}

The authors declare that their contributions are equal. All authors read and approved the final manuscript.

\section{Author details}

${ }^{1}$ Department of Mathematics, National Institute of Technology Rourkela, Rourkela 769008, Odisha, India. ${ }^{2}$ Faculty of Education and Faculty of Mathematics and Physics, University of Ljubljana, Ljubljana, 1000, Slovenia.

\section{Publisher's Note}

Springer Nature remains neutral with regard to jurisdictional claims in published maps and institutional affiliations.

Received: 31 May 2021 Accepted: 27 September 2021 Published online: 18 October 2021

\section{References}

1. Abdellaoui, B., Bentifour, R.: Caffarelli-Kohn-Nirenberg type inequalities of fractional order and applications. J. Funct. Anal. 272(10), 3998-4029 (2017)

2. Adams, R.A., Fournier, J.J.F.: Sobolev Spaces. Elsevier, Oxford (2014)

3. Alves, C.O., Figueiredo, G.M.: Existence of positive solution for a planar Schrödinger-Poisson system with exponential growth. J. Math. Phys. 60(1), Article ID 011503 (2019)

4. Autuori, G., Pucci, P.: Elliptic problems involving the fractional Laplacian in $R^{N}$. J. Differ. Equ. 255(8), $2340-2362$ (2013)

5. Biswas, R., Tiwari, S.: Variable order nonlocal Choquard problem with variable exponents. Complex Var. Elliptic Equ. 66(5), 853-875 (2021)

6. Boër, E. de S., Miyagaki, O.H.: The Choquard logarithmic equation involving fractional Laplacian operator and a nonlinearity with exponential critical growth (2020). 2011.12806v2 [math.AP]

7. Bonheure, D., Cingolani, S., Van Schaftingen, J.: The logarithmic Choquard equation: sharp asymptotics and nondegeneracy of the ground state. J. Funct. Anal. 272(12), 5255-5281 (2017)

8. Caffarelli, L.: Non-local diffusions, drifts and games. In: Holden, H., Karlsen, K. (eds.) Nonlinear Partial Differential Equations. Abel Symposia, vol. 7, pp. 37-52. Springer, Berlin (2012) 
9. Cao, D.M.: Nontrivial solution of semilinear elliptic equation with critical exponent in $\mathbb{R}^{2}$. Commun. Partial Differ. Equ. 17(3-4), 407-435 (1992)

10. Chang, X., Wang, Z.Q.: Ground state of scalar field equations involving a fractional Laplacian with general nonlinearity. Nonlinearity 26(2), 479-494 (2013)

11. Choudhuri, D.: Existence and Hölder regularity of infinitely many solutions to a $p$-Kirchhoff-type problem involving a singular nonlinearity without the Ambrosetti-Rabinowitz (AR) condition. Z. Angew. Math. Phys. 72(1), 36 (2021)

12. Choudhuri, D., Saoudi, K.: Elliptic problem in an exterior domain driven by a singularity with a nonlocal Neumann condition (2021). arXiv:2012.04449

13. Cingolani, S., Jeanjean, L.: Stationary waves with prescribed $L^{2}$-norm for the planar Schrödinger-Poisson system. SIAM J. Math. Anal. 51(4), 3533-3568 (2019)

14. Cingolani, S., Weth, T.: On the planar Schrödinger-Poisson system. Ann. Inst. Henri Poincaré, Anal. Non Linéaire 33(1), 169-197 (2016)

15. Di Nezza, E., Palatucci, G., Valdinoci, E.: Hitchhiker's guide to the fractional Sobolev spaces. Bull. Sci. Math. 136(5), 521-573 (2012)

16. Dipierro, S., Palatucci, G., Valdinoci, E.: Dislocation dynamics in crystals: a macroscopic theory in a fractional Laplace setting. Commun. Math. Phys. 333(2), 1061-1105 (2015)

17. do Ó, J.M., Miyagaki, O.H., Squassina, M.: Nonautonomous fractional problems with exponential growth. NoDEA Nonlinear Differ. Equ. Appl. 22(5), 1395-1410 (2015)

18. do Ó, J.M., Miyagaki, O.H., Squassina, M.: Critical and subcritical fractional problems with vanishing potentials. Commun. Contemp. Math. 18(6), 1550063 (2016)

19. do Ó, J.M., Miyagaki, O.H., Squassina, M.: Ground states of nonlocal scalar field equations with Trudinger-Moser critical nonlinearity. Topol. Methods Nonlinear Anal. 48(2), 477-492 (2016)

20. Du, M., Weth, T.: Ground states and high energy solutions of the planar Schrödinger-Poisson system. Nonlinearity 30(9), 3492-3515 (2017)

21. Felmer, P., Quaas, A., Tan, J.: Positive solutions of the nonlinear Schrödinger equation with the fractional Laplacian. Proc. R. Soc. Edinb., Sect. A 142(6), 1237-1262 (2012)

22. Ghanmi, A., Saoudi, K.: A multiplicity result for a singular problem involving the fractional $p$-Laplacian operator. Complex Var. Elliptic Equ. 61(9), 1199-1216 (2016)

23. Goel, D., Rădulescu, V., Sreenadh, K.: Coron problem for nonlocal equations involving Choquard nonlinearity. Adv. Nonlinear Stud. 20(1), 141-161 (2020)

24. Guo, Q., Wu, J.: Existence of solutions to the logarithmic Choquard equations in high dimensions. Bull. Malays. Math. Sci. Soc. 43(2), 1545-1553 (2020)

25. Iannizzotto, A., Squassina, M.: Weyl-type laws for fractional $p$-eigenvalue problems. Asymptot. Anal. 88(4), 233-245 (2014)

26. Lam, N., Lu, G.: Elliptic equations and systems with subcritical and critical exponential growth without the Ambrosetti-Rabinowitz condition. J. Geom. Anal. 24(1), 118-143 (2014)

27. Lazer, A.C., McKenna, P.J.: On a singular nonlinear elliptic boundary-value problem. Proc. Am. Math. Soc. 111(3), 721-730 (1991)

28. Lieb, E.H.: Existence and uniqueness of the minimizing solution of Choquard's nonlinear equation. Stud. Appl. Math. 57(2), 93-105 (1977)

29. Lieb, E.H.: Sharp constants in the Hardy-Littlewood-Sobolev and related inequalities. Ann. Math. 118(2), 349-374 (1983)

30. Moser, J.: A sharp form of an inequality by N. Trudinger. Indiana Univ. Math. J. 20(11), 1077-1092 (1971)

31. Oliva, F., Petitta, F.: On singular elliptic equations with measure sources. ESAIM Control Optim. Calc. Var. 22(1), 289-308 (2016)

32. Ozawa, T:: On critical cases of Sobolev's inequalities. J. Funct. Anal. 127(2), 259-269 (1995)

33. Panda, A., Choudhuri, D., Saoudi, K.: A critical fractional Choquard problem involving a singular nonlinearity and a Radon measure. J. Pseudo-Differ. Oper. Appl. 12(1), 22 (2021)

34. Papageorgiou, N.S., Rădulescu, V.D., Repovš, D.D.: Nonlinear Analysis - Theory and Methods. Springer Monographs in Mathematics. Springer, Cham (2019)

35. Pucci, P., Xiang, M., Zhang, B.: Multiple solutions for nonhomogeneous Schrödinger-Kirchhoff type equations involving the fractional $p$-Laplacian in $\mathbb{R}^{N}$. Calc. Var. Partial Differ. Equ. 54(3), 2785-2806 (2015)

36. Ros-Oton, X., Serra, J.: The Pohozaev identity for the fractional Laplacian. Arch. Ration. Mech. Anal. 213(2), 587-628 (2014)

37. Saoudi, K., Ghosh, S., Choudhuri, D.: Multiplicity and Hölder regularity of solutions for a nonlocal elliptic PDE involving singularity. J. Math. Phys. 60(10), Article ID 101509 (2019)

38. Schechter, M.: The use of cerami sequences in critical point theory. Abstr. Appl. Anal. 2007, Article ID 58948 (2007)

39. Soni, A., Choudhuri, D.: Existence of multiple solutions to an elliptic problem with measure data. J. Elliptic Parabolic Equ. 4(2), 369-388 (2018)

40. Stubbe, J.: Bound states of two-dimensional Schrödinger-Newton equations. 0807.4059 [math-ph]

41. Wen, L., Tang, X., Chen, S.: Ground state solutions to logarithmic Choquard equations in $\mathbb{R}^{3}$. Math. Methods Appl. Sci. 43(7), 4222-4238 (2020)

42. Yu, M., Zhang, M., Zhang, X.: Fractional minimization problem on the Nehari manifold. Electron. J. Differ. Equ. 2018, Article ID 82 (2018) 